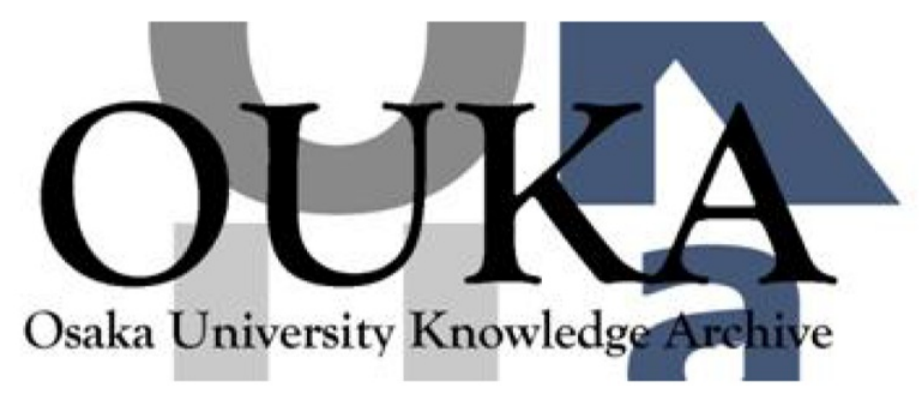

\begin{tabular}{|c|l|}
\hline Title & $\begin{array}{l}\text { High Q defect mode and laser action in one- } \\
\text { dimensional hybrid photonic crystal containing } \\
\text { cholesteric liquid crystal }\end{array}$ \\
\hline Author(s) & $\begin{array}{l}\text { Matsuhisa, Yuko; Ozaki, Ryotaro; Yoshino, } \\
\text { Katsumi et al. }\end{array}$ \\
\hline Citation & $\begin{array}{l}\text { Applied Physics Letters. 89(10) p. 101109- } \\
\text { p. } 101109\end{array}$ \\
\hline Issue Date & $2006-09-06$ \\
\hline oaire:version & VoR \\
\hline URL & https://hdl. handle.net/11094/75826 \\
\hline rights & \\
\hline Note & \\
\hline
\end{tabular}

Osaka University Knowledge Archive : OUKA

https://ir. Library. osaka-u. ac. jp/

Osaka University 


\section{High $Q$ defect mode and laser action in one-dimensional hybrid photonic crystal containing cholesteric liquid crystal}

Cite as: Appl. Phys. Lett. 89, 101109 (2006); https://doi.org/10.1063/1.2347114

Submitted: 16 December 2005 . Accepted: 22 July 2006 . Published Online: 06 September 2006

Yuko Matsuhisa, Ryotaro Ozaki, Katsumi Yoshino, and Masanori Ozaki

\section{ARTICLES YOU MAY BE INTERESTED IN}

Defect mode lasing from a double-layered dye-doped polymeric cholesteric liquid crystal films with a thin rubbed defect layer

Applied Physics Letters 90, 261108 (2007); https://doi.org/10.1063/1.2752017

Electrically color-tunable defect mode lasing in one-dimensional photonic-band-gap system containing liquid crystal

Applied Physics Letters 82, 3593 (2003); https://doi.org/10.1063/1.1577829

Linearly polarized lasing in one-dimensional hybrid photonic crystal containing cholesteric liquid crystal

Journal of Applied Physics 101, 033120 (2007); https://doi.org/10.1063/1.2434835

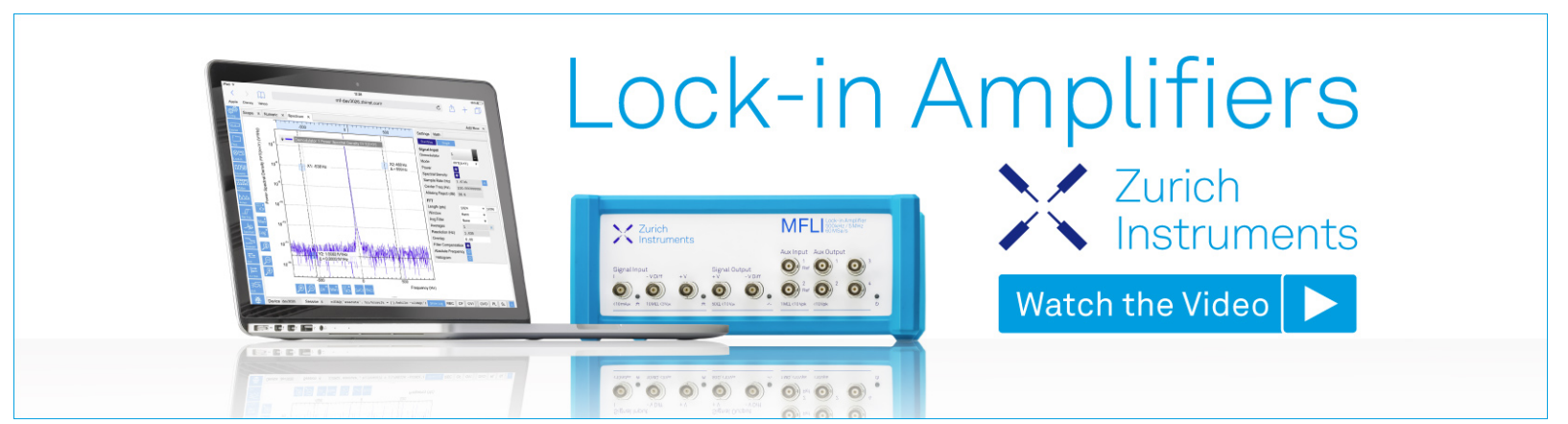




\title{
High $Q$ defect mode and laser action in one-dimensional hybrid photonic crystal containing cholesteric liquid crystal
}

\author{
Yuko Matsuhisa and Ryotaro Ozaki ${ }^{\text {a) }}$ \\ Department of Electrical, Electronic and Information Engineering, Graduate School of Engineering, \\ Osaka University, 2-1 Yamada-oka, Suita, Osaka 565-0871, Japan \\ Katsumi Yoshino \\ Center for University-Industry Cooperation, Shimane University, Matsue, Shimane 690-0816, Japan \\ and Innovation Center for Advanced Science, Osaka University, Yamada-oka 2-1, Suita, \\ Osaka 565-0871, Japan

\begin{abstract}
Masanori Ozaki
Department of Electrical, Electronic and Information Engineering, Graduate School of Engineering, Osaka University, 2-1 Yamada-oka, Suita, Osaka 565-0871, Japan
\end{abstract}

(Received 16 December 2005; accepted 22 July 2006; published online 6 September 2006)

\begin{abstract}
The authors have investigated the optical characteristics of a one-dimensional hybrid photonic crystal (1D HPC) containing cholesteric liquid crystal (CLC) as a defect by theoretical calculation and predicted the appearance of additional modes at the band edges of the CLC defect, whose $Q$ factor was higher than those of the other defect modes. They have confirmed the appearance of the additional mode experimentally. Single-mode laser action with low pumping threshold was observed in a 1D HPC with a dye-doped CLC defect, which is based on the additional defect mode with a high $Q$ factor peculiar to the CLC defect having periodic structure. (C) 2006 American Institute of Physics. [DOI: 10.1063/1.2347114]
\end{abstract}

A photonic crystal (PC) has a periodic dielectric structure with a periodicity in the range of optical wavelengths. In a PC, the propagation of light is inhibited in a certain energy range of photons, which is called a photonic band gap (PBG), and photon group velocity is suppressed at the edge of the PBG. ${ }^{1}$ Cholesteric liquid crystals (CLCs) and chiral smectic liquid crystals have chirality in their molecular structure and spontaneously form one-dimensional (1D) periodic helical structures, which can be regarded as 1D PCs. Lasing at the band edge has been reported for such liquid crystals. ${ }^{2-8}$ On the other hand, photons are localized upon the introduction of a defect in a PC. ${ }^{9}$ By utilizing the photon localization, various applications are expected, such as low-threshold lasers and microwaveguides. ${ }^{0-13}$

We have, so far, investigated hybrid photonic crystals (HPCs) that are fabricated from inorganic PC and liquid crystals, and proposed tunable PCs. ${ }^{14-21}$ Recently, we have reported a HPC based on the combination of CLC and a PC consisting of $\mathrm{SiO}_{2}$ and $\mathrm{TiO}_{2}$ multilayers, and achieved single-mode laser action. ${ }^{21}$ In this system, the lasing threshold was much lower than that in simple CLC without the PC. However, the details of the mechanism of lowering the lasing threshold were not clear. In this study, we have both theoretically and experimentally investigated the optical characteristics of a 1D HPC containing CLC as a defect, and laser action in a HPC with a dye-doped CLC defect.

Dielectric multilayers, each consisting of five pairs of alternately stacked $\mathrm{SiO}_{2}$ and $\mathrm{TiO}_{2}$ layers deposited on a glass substrate, were used on both sides of a CLC. The thicknesses of the $\mathrm{SiO}_{2}$ and $\mathrm{TiO}_{2}$ layers were 111 and $69 \mathrm{~nm}$, respectively. The top surface of a dielectric multilayer was coated with polyimide (JSR, AL1254) and unidirectionally rubbed in the direction in which the helix axis is perpendicular to the

\footnotetext{
${ }^{a)}$ Electronic mail: ozaki@opal.eei.eng.osaka-u.ac.jp
}

substrates. The CLC host was prepared by mixing using a chiral center (Merck, S-811) and a nematic liquid crystal (Merck, E44), which was induced between dielectric multilayers with $9.1 \mu \mathrm{m}$ spacing. The extraordinary and ordinary refractive indices of the mixture were 1.71 and 1.54, respectively.

We have theoretically calculated the transmission spectra of a 1D HPC with a CLC defect. Figure 1(a) shows the transmission spectrum of a ten-pair multilayer without a CLC defect (solid line) and a CLC without a PC structure (dashed line). The PBG of the CLC was observed between 605 and $680 \mathrm{~nm}$, which is inside that of the multilayer. Fig-

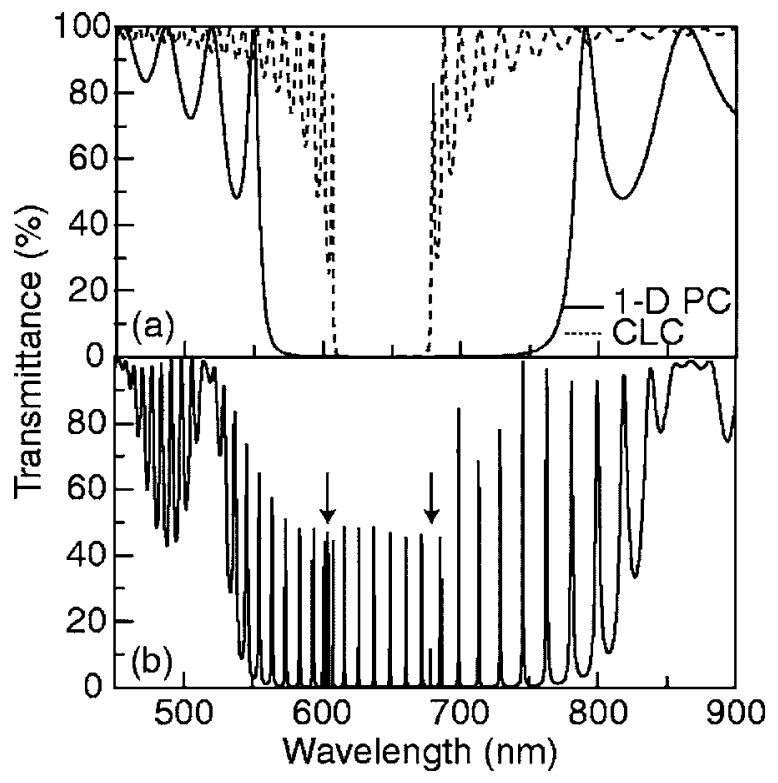

FIG. 1. (a) Calculated transmission spectra of 1D PC without defect and CLC. (b) Calculated transmission spectrum of 1D HPC with CLC defect. 


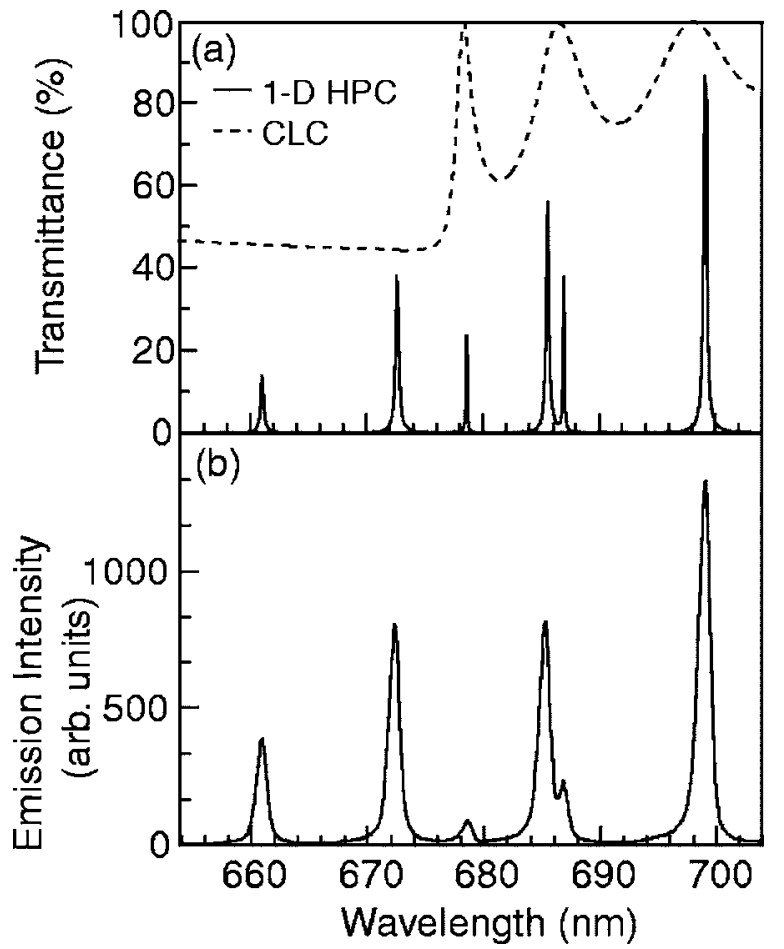

FIG. 2. (a) Calculated transmission spectra of $1 D$ HPC with CLC defect and CLC. (b) Experimental transmission spectrum of 1D HPC with CLC defect.

ure 1(b) shows the calculated transmission spectrum of a 1D HPC with a CLC defect. Many peaks appeared at regular intervals in the PBG of the HPC. These peaks are related to the defect modes resulting from the introduction of the CLC defect. However, additional peaks were observed, as indicated by arrows, which disrupted the regular interval between the defect mode peaks at both band edges of the CLC.

The transmission spectra in Fig. 1, around the longer edge of the PBG of the CLC, are shown magnified in Fig. 2(a). Four main peaks due to the defect modes appeared at regular intervals $(661,673,687$, and $699 \mathrm{~nm}$ ), although the peak at $687 \mathrm{~nm}$ splits. The splitting of the peak at $687 \mathrm{~nm}$ is attributed to the optical anisotropy of the CLC. Therefore, two kinds of defect mode corresponding to left- and righthanded circularly polarized lights could exist out of the PBG of the CLC. On the other hand, one additional peak was observed at $678.6 \mathrm{~nm}$, which corresponds to the band-edge wavelength of the CLC. From detailed consideration of the polarization states of transmitted light, the additional peak was clearly distinguished from the other defect mode peaks. Such a peak was not observed in a 1D PC with a uniform defect such as an isotropic medium or nematic liquid crystals. ${ }^{17-19}$ Namely, this peak is a defect mode peculiar to the helix defect in the 1D PC, and is associated with photon localization originating from the band-edge effect of the CLC helix. Note that this defect mode peak is very sharp and the full width at half maximum (FWHM) of this peak was $0.05 \mathrm{~nm}$, which is more than four times smaller than that of the other defect mode peaks $(0.23 \mathrm{~nm})$. From the peak width, the $Q$ factor of the additional mode at the band edge of the CLC was estimated to be 14000 , which was much higher than that of the other defect modes.

To confirm the appearance of the peculiar defect mode, the transmission spectrum of a 1D HPC with a CLC defect was experimentally measured by detecting the light passing

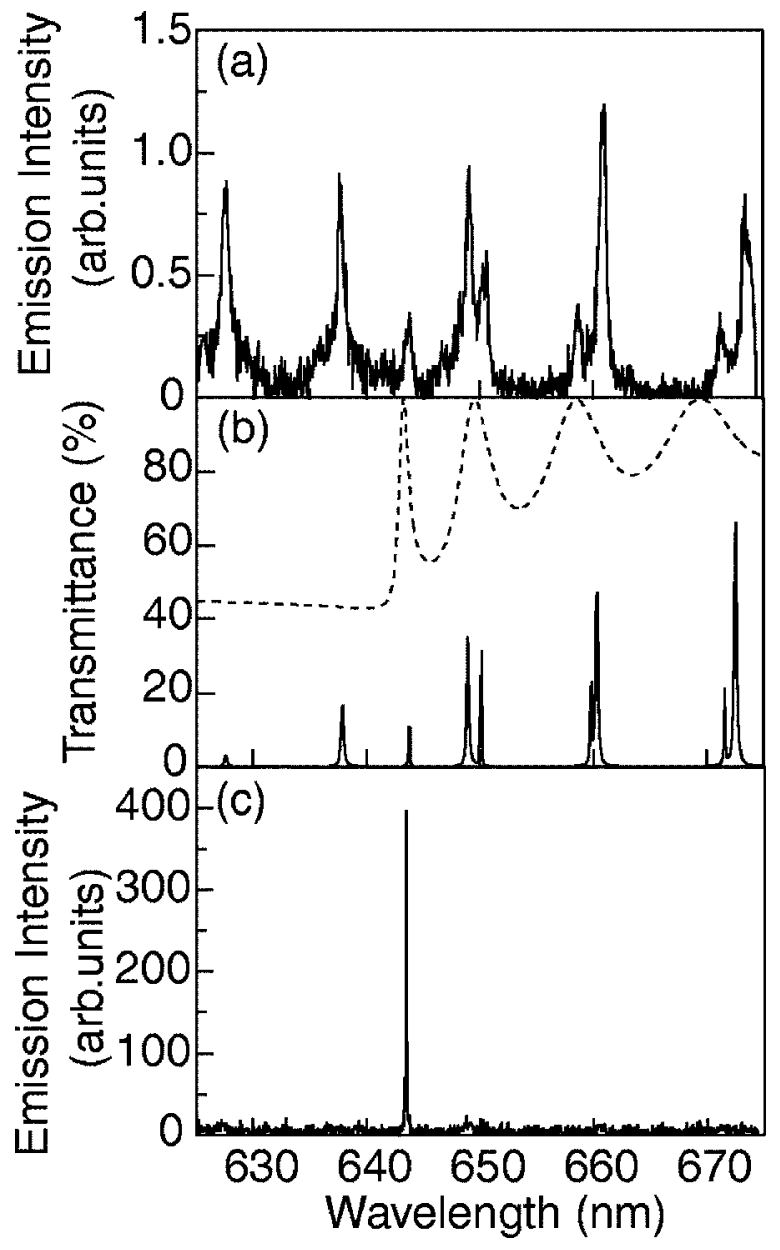

FIG. 3. (a) Emission spectrum of 1D HPC with CLC defect at pumping energy of $9.0 \mathrm{~nJ} /$ pulse. (b) Calculated transmission spectra of 1D HPC with CLC defect and CLC. (c) Emission spectrum of 1D HPC with CLC defect at pumping energy of $18 \mathrm{~nJ} /$ pulse.

through the HPC cell using a multichannel spectrometer (Oriel, MS257) having a spectral resolution of $0.2 \mathrm{~nm}$. Figure 2(b) shows the transmission spectrum of the 1D HPC with a CLC defect. It is in good agreement with the calculated result shown in Fig. 2(a). However, no significant difference in FWHM among the defect mode peaks was observed because of the experimentally limited spectral resolution and the spatial uniformity of the HPC cell. From the result, we have both experimentally and theoretically confirmed the existence of a peculiar peak with a high $Q$ factor at the band edge of a CLC in a 1D HPC with a CLC defect. Note that this peculiar defect mode with a high $Q$ factor exists necessarily under any set of conditions as long as the PBG of the CLC is inside that of the multilayer.

We have investigated the laser action in a 1D HPC with a CLC defect. As a laser dye dopant, [2-[24(dimethylamino)pheny1]etheny 1]-6-methy1-4H-pyran-4ylidene propanedinitrile (Exciton) was compounded in a CLC, whose concentration was $1 \mathrm{wt} \%$. The PBG of the CLC shifts with a temperature change, which is attributed to the temperature dependence of the helical pitch of the CLC. The band edge of the CLC was adjusted to $644 \mathrm{~nm}$ by temperature regulation in this experiment because of the emission wavelength window of the laser dye. The second harmonic light of a $Q$-switch neodymium-doped yttrium aluminum garnet laser (Spectra Physics, Quanta-Ray INDI), 
whose wavelength, pulse width, and pulse repetition frequency were $532 \mathrm{~nm}, 8 \mathrm{~ns}$, and $10 \mathrm{~Hz}$, respectively, was used for the excitation. The excitation laser beam irradiated the sample perpendicularly to the glass surface, whose illumination area on the sample was about $0.2 \mathrm{~mm}^{2}$. The emission spectra from the 1D HPC were measured using a charge-coupled device multichannel spectrometer.

Figure 3(a) shows the emission spectrum of a 1D HPC with a dye-doped CLC defect at the pumping energy of $9.0 \mathrm{~nJ} /$ pulse. The emission peaks were attributed to spontaneously emitted light passing out through narrow spectral windows owing to the defect modes. Mainly five defect modes appeared, at regular intervals, although three peaks split at longer wavelengths $(>645 \mathrm{~nm})$. A peculiar defect mode was observed at $643.5 \mathrm{~nm}$, which corresponds to the band-edge wavelength of the CLC. We calculated the transmission spectrum of this system, as shown in Fig. 3(b). We observed the peculiar defect mode with a high $Q$ factor at $643.5 \mathrm{~nm}$, which was in good agreement with experimental result shown in Fig. 3(a).

At a high pumping energy of $18 \mathrm{~nJ} /$ pulse, as shown in Fig. 3(c), only one sharp lasing peak appeared at $643.5 \mathrm{~nm}$, which coincides with the wavelength of the peculiar defect mode. Note that the laser action was single-mode based on one additional mode, although many modes exist because of the high $Q$ factor. The threshold of laser action in the 1D HPC with a CLC defect was lower than that in simple CLC without a 1D PC. ${ }^{21}$ This result is attributed to strong optical confinement due to the high $Q$ factor of the additional mode.

In conclusion, we theoretically calculated the transmission spectra of a 1D HPC with a CLC defect, and found one additional peculiar mode with a high $Q$ factor at the band edge of the CLC. We also confirmed the additional mode experimentally. Optically pumped laser action was observed in a 1D HPC with a dye-doped CLC defect. Single-mode laser action was observed that was based on the peculiar defect mode with a high $Q$ factor.
This work is partially supported by a Grant-in-Aid for Scientific Research from the Ministry of Education, Culture, Sports, Science and Technology of Japan.

${ }^{1}$ E. Yablonovitch, Phys. Rev. Lett. 58, 2059 (1987).

${ }^{2}$ V. I. Kopp, B. Fan, H. K. M. Vithana, and A. Z. Genack, Opt. Lett. 23, 1707 (1998)

${ }^{3}$ B. Taheri, A. F. Munoz, P. Palffy-Muhoray, and R. Twieg, Mol. Cryst. Liq. Cryst. Sci. Technol., Sect. A 358, 73 (2001).

${ }^{4}$ A. F. Munoz, P. Palffy-Muhoray, and B. Taheri, Opt. Lett. 26, 804 (2001).

${ }^{5}$ T. Matsui, R. Ozaki, K. Funamoto, M. Ozaki, and K. Yoshino, Appl. Phys. Lett. 81, 3741 (2002)

${ }^{6}$ K. Funamoto, M. Ozaki, and K. Yoshino, Jpn. J. Appl. Phys., Part 2 42, L1523 (2003).

${ }^{7}$ M. Ozaki, M. Kasano, D. Ganzke, W. Haase, and K. Yoshino, Adv. Mater. (Weinheim, Ger.) 14, 306 (2002).

${ }^{8}$ M. Kasano, M. Ozaki, K. Yoshino, D. Ganzke, and W. Haase, Appl. Phys. Lett. 82, 4026 (2003).

${ }^{9}$ S. John, Phys. Rev. Lett. 58, 2486 (1987).

${ }^{10}$ J. S. Foresi, P. R. Villeneuve, J. Ferrera, E. R. Thoen, G. Steinmeyer, S. Fan, J. D. Joannopoulos, L. C. Kimerling, H. I. Smith, and E. P. Ippen, Nature (London) 390, 143 (1997).

${ }^{11}$ O. Painter, R. K. Lee, A. Scherer, A. Yariv, J. D. O’Brien, P. D. Dapkus, and I. Kim, Science 284, 1819 (1999).

${ }^{12}$ S. Noda, A. Chutinan, and M. Imada, Nature (London) 407, 608 (2000).

${ }^{13}$ T. Hattori, N. Tsurumachi, and H. Nakatsuka, J. Opt. Soc. Am. B 14, 348 (1997).

${ }^{14}$ K. Yoshino, S. Satoh, Y. Shimoda, Y. Kawagishi, K. Nakayama, and M. Ozaki, Jpn. J. Appl. Phys., Part 1 38, 961 (1999).

${ }^{15}$ K. Yoshino, Y. Shimoda, Y. Kawagishi, K. Nakayama, and M. Ozaki, Appl. Phys. Lett. 75, 932 (1999).

${ }^{16}$ Y. Shimoda, M. Ozaki, and K. Yoshino, Appl. Phys. Lett. 79, 3627 (2001).

${ }^{17}$ R. Ozaki, T. Matsui, M. Ozaki, and K. Yoshino, Jpn. J. Appl. Phys., Part 2 41, L1482 (2002).

${ }^{18}$ R. Ozaki, T. Matsui, M. Ozaki, and K. Yoshino, Appl. Phys. Lett. 82, 3593 (2003).

${ }^{19}$ R. Ozaki, M. Ozaki, and K. Yoshino, Jpn. J. Appl. Phys., Part 2 42, L669 (2003).

${ }^{20}$ R. Ozaki, Y. Matsuhisa, M. Ozaki, and K. Yoshino, Appl. Phys. Lett. 84, 1844 (2004).

${ }^{21}$ Y. Matsuhisa, R. Ozaki, M. Ozaki, and K. Yoshino, Jpn. J. Appl. Phys., Part 2 44, L629 (2005). 